\title{
Analysis of vertical wave number spectrum of atmospheric gravity waves in the stratosphere using COSMIC GPS radio occultation data
}

\author{
T. Tsuda ${ }^{1}$, X. Lin ${ }^{1,}{ }^{*}$, H. Hayashi ${ }^{1}$, and Noersomadi ${ }^{2}$ \\ ${ }^{1}$ Research Institute for Sustainable Humanosphere (RISH), Kyoto University, Uji, Kyoto, Japan \\ ${ }^{2}$ National Institute of Aeronautics and Space (LAPAN), Bandung, Indonesia \\ *now at: KDDI Co. Ltd., Tokyo, Japan
}

Received: 25 February 2011 - Published in Atmos. Meas. Tech. Discuss.: 4 April 2011

Revised: 21 July 2011 - Accepted: 7 August 2011 - Published: 26 August 2011

\begin{abstract}
GPS radio occultation (RO) is characterized by high accuracy and excellent height resolution, which has great advantages in analyzing atmospheric structures including small-scale vertical fluctuations. The vertical resolution of the geometrical optics (GO) method in the stratosphere is about $1.5 \mathrm{~km}$ due to Fresnel radius limitations, but full spectrum inversion (FSI) can provide superior resolutions. We applied FSI to COSMIC GPS-RO profiles from ground level up to $30 \mathrm{~km}$ altitude, although basic retrieval at UCAR/CDAAC sets the sewing height from GO to FSI below the tropopause. We validated FSI temperature profiles with routine high-resolution radiosonde data in Malaysia and North America collected within $400 \mathrm{~km}$ and about $30 \mathrm{~min}$ of the GPS RO events. The average discrepancy at $10-30 \mathrm{~km}$ altitude was less than $0.5 \mathrm{~K}$, and the bias was equivalent with the GO results.
\end{abstract}

Using the FSI results, we analyzed the vertical wave number spectrum of normalized temperature fluctuations in the stratosphere at $20-30 \mathrm{~km}$ altitude, which exhibits good consistency with the model spectra of saturated gravity waves. We investigated the white noise floor that tends to appear at high wave numbers, and the substantial vertical resolution of the FSI method was estimated as about $100-200 \mathrm{~m}$ in the lower stratosphere. We also examined a criterion for the upper limit of the FSI profiles, beyond which bending angle perturbations due to system noises, etc., could exceed atmospheric excess phase fluctuations. We found that the FSI profiles can be used up to about $28 \mathrm{~km}$ in studies of temperature fluctuations with vertical wave lengths as short as $0.5 \mathrm{~km}$.

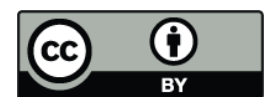

Correspondence to: $\mathrm{T}$. Tsuda (tsuda@ rish.kyoto-u.ac.jp)

\section{Introduction}

The Global Positioning System (GPS) radio occultation (GPS-RO) technique is an active limb sounding observation of the Earth's atmosphere using a GPS receiver onboard a low Earth orbit (LEO) satellite. The first GPS RO mission, GPS/MET (GPS/Meteorology) (Ware et al., 1996), was successfully conducted by the University Corporation for Atmospheric Research (UCAR) in collaboration with the Jet Propulsion Laboratory (JPL) in 1995 using a micro-satellite, MicroLab-1. Since this pioneering experiment a number of GPS-RO missions have been carried out, such as the Challenging Minisatellite Payload (CHAMP) (Wickert et al., 2001) in 2001, Satellite de Aplicaciones Cientificas-C (SACC) in 2001, and the Gravity Recovery and Climate Experiment (GRACE) in 2002. In 2006 the Constellation Observing System for Meteorology, Ionosphere and Climate (FORMOSAT3/COSMIC) (Anthes et al., 2008) was launched as a joint project between UCAR and the National Space Organization (NSPO) of Taiwan. The GPS-RO has become an important measurement technique, greatly impacting diverse applications in the atmospheric sciences, meteorology, boundary layer studies, numerical weather prediction, global climate change, and ionospheric disturbances.

With a single LEO satellite, about 250 GPS-RO events can be obtained per day using a single occultation antenna. The COSMIC mission employs a constellation of six LEO satellites having two forward and backward antennas on each satellite, for a total of about 2500 GPS-RO data points per day. Because the GPS RO is a limb sounding technique, it has an inherent advantage in height resolution. Further, by applying advanced radio holographic ( $\mathrm{RH})$ methods, vertical resolution was as good as a few hundred meters in the lower

Published by Copernicus Publications on behalf of the European Geosciences Union. 
atmosphere. Such a good height resolution, comparable to a ground-based radiosonde, has not been attained with other conventional satellite techniques.

Most current retrieval techniques for GPS RO assume spherical symmetry of the atmosphere in their inversion process, so the horizontal resolution of a retrieved profile centered at a tangent point is roughly $300 \mathrm{~km}$. Note also that the tangent point of the ray drifts horizontally. Knowledge on spatial variations of the atmospheric field can be gained, however, when the full radio path is used by applying an advanced technique, such as a non-local parameter for assimilation into a mesoscale numerical weather prediction model (e.g. Seko et al., 2009).

There are two basic inversion algorithms for GPS RO, geometrical optics (GO) and radio holography (RH). Various techniques have been proposed for RH. Among these, in this study we use the Full Spectrum Inversion (FSI) method (Jensen et al., 2003). Both GO and FSI are employed at the COSMIC Data Analysis and Archive Center (CDAAC) of UCAR. At the CDAAC, GO is normally applied from the top of a profile down to the upper troposphere, and then the retrieval procedure is switched to FSI from there to the ground.

In this study, we primarily follow the software system at CDAAC, but with modification of the height range for FSI analysis so as to obtain a high-resolution temperature profile in the stratosphere. We validate the accuracy and vertical resolution of FSI retrieval in the stratosphere through comparison with radiosondes. Taking advantage of the increased height resolution, we study the vertical wave number spectra of temperature perturbations at $20-30 \mathrm{~km}$, and discuss consistency with theoretical predictions of saturated gravity waves. We also analyze the valid range of the FSI retrievals.

Atmospheric gravity wave activity in the stratosphere is now understood to occur nearly continuously regardless of season, location and altitude, although significant variations exist depending on wave generation mechanisms, wavemean flow interactions, and so on. During early stage of the COSMIC mission, pairs of LEO satellites performed a tandem flight on the same orbit. A pair of nearly simultaneous GPS RO profiles with short spatial separation provided a unique opportunity to confirm that small vertical scale temperature fluctuations can be attributed to real geophysical variability, such as gravity waves (Horinouchi and Tsuda, 2009; McDonald et al., 2010).

GPS RO profiles normally appear as a projection of many gravity waves with different frequency, vertical and horizontal wavenumbers. Statistical characteristics of such perturbations can be described well by analyzing a vertical wavenumber spectrum. It is noteworthy, however, that the horizontal resolution of GPS RO is about $300 \mathrm{~km}$, being common to a limb scanning method (e.g. Preusse et al., 2008). Because of this observational filter effect (Alexander, 1998), a part of the horizontal wavenumber spectrum is removed. Assuming a horizontal wavenumber spectrum with a logarithmic slope of $-5 / 3$ extending from $1 \mathrm{~km}$ to $5000 \mathrm{~km}$ in wavelengths, Tsuda et al. (2000) estimated the reduction of the estimated wave energy to be less than $15 \%$. GPS RO can resolve most of the vertical wave number spectrum with wavelengths ranging from a few hundred meters to about $10 \mathrm{~km}$, which is very unique compared to other satellite instruments.

\section{Retrieval of GPS RO profiles}

\subsection{Geometrical optics (GO)}

Here we briefly introduce the fundamentals of the GPS RO retrieval method. The necessary information for retrieval is the amplitude and phase of the occulted GPS radio signals. Based on the precise orbit information of the GPS and LEO satellite, the atmospheric excess phase (delay) is calculated.

The GO method was successfully applied to GPS/MET, and the accuracy of the retrieved temperature is statistically estimated as within $1 \mathrm{~K}$ (Kursinski et al., 1996). GO is an approximated method of the electromagnetic waves obtained by considering Maxwell's equations in the limiting case where the vacuum wave length limits to 0 . Because the wave lengths of the two GPS signals, L1 and L2, are on the order of $20 \mathrm{~cm}$, comparing these with the scale of the atmospheric phenomena indicates that this hypothesis is a good assumption. But this also implies that the GO method is not valid at shadow boundaries and caustics due to their nonmonotonic refractive gradients (e.g. Melbourne, 2004).

The first retrieved result is a bending angle profile of the L1 and L2 signals. The received signal is smoothed, with the normal filter for the L1 signal corresponding to a height smoothing of about $0.8-1 \mathrm{~km}$. Because of its poorer quality, however, the L2 signal may not provide a bending angle profile with the same precision and resolution as the L1 signal. A longer smoothing window is therefore sometimes applied to reduce the effect of L2 noise, giving a height smearing over $3-4 \mathrm{~km}$. Ionospheric calibration then eliminates the ionospheric effects using the $\mathrm{L} 1$ and $\mathrm{L} 2$ bending angles. Note that small-scale ionosphere perturbations may still remain as retrieval errors after ionospheric calibration.

In altitudes higher than about $40 \mathrm{~km}$, the magnitude of the neutral atmospheric bending angle becomes very small due to its exponential decrease, sometimes making it impossible to extract a useful signal because the magnitude of receiver system noise remains constant. The uncertainty of the signal at high altitude could introduce a bias that propagates toward lower altitudes through Abel inversion. To reduce this effect, the observed bending angle is subjected to optimal estimation by referring to a bending angle from climate models.

An Abel inversion transforms the optimized bending angle profile to a refractive index profile under the basic hypothesis that the atmosphere is spherically symmetric. Pressure and temperature profiles can be derived from the refractive index. Note that in a moist atmosphere the refractive index cannot be uniquely converted to a temperature, so the contribution 
of water vapor to the refractive index must be separated out by using, for example, a variational method with a numerical weather forecast as its initial value.

The available height range of the GPS-RO profiles is normally below about $40 \mathrm{~km}$. Above $40 \mathrm{~km}$, ionospheric effects and other measurement errors could exceed the atmospheric phase delay, which decreases exponentially with height. On the other hand, at the lowest part of the troposphere multipath effects become a serious problem because of a sharp height gradient of the refractive index due to water vapor. Moreover, below the tropopause the horizontally irregular distribution of water vapor may invalidate the hypothesis of a spherically symmetric atmosphere, as is assumed at higher altitudes. This will also introduce errors to retrieved results.

Kursinski et al. (1997) estimated accuracy in the temperature inversion as $0.2-0.4 \mathrm{~K}$ at $5-30 \mathrm{~km}$. Comparing nearby GPS RO profiles with CHAMP and SAC-C, Hajj et al. (2004) evaluated discrepancy to less than $0.86 \mathrm{~K}$ in the interval between 5 and $15 \mathrm{~km}$. Because the GO method describes electromagnetic waves as optical rays, vertical resolution is limited by Fresnel diffraction. The vertical width of the first Fresnel zone is on average about $1.5 \mathrm{~km}$ in the stratosphere, and roughly $0.5 \mathrm{~km}$ in the lower troposphere (e.g. Melbourne, 2004).

\subsection{Full Spectral Inversion (FSI)}

Although GO has reasonably good vertical resolution, it is limited by the Fresnel zone. Moreover, GO cannot overcome the multipath problem in the complex atmospheric structures frequently caused by water vapor in the lower troposphere. To solve the multipath problem and obtain a better vertical resolution, radio holographic ( $\mathrm{RH})$ methods are introduced to the GPS RO retrieval. The RH methods are types of wave optical methods that invert the occulted radio signal in a complex form. At CDAAC several RH methods are used as an inversion algorithm, including back propagation (BP) (Gorbunov et al., 1996; Karayel and Hinson, 1997), sliding spectrum (SS) (Sokolovskiy, 2001), canonical transform (CT) (Gorbunov et al., 2002), and full spectrum inversion (FSI) (Jensen et al., 2003). Among these RH methods FSI provides the same accuracy and resolution to BP and CT. Moreover FFT can also be used by assuming that the GPS and LEO satellites are in a circular orbit, considerably reducing computational cost. The FSI method is therefore widely used.

In the standard retrieval program at CDAAC, the FSI method only retrieves the L1C signal below some sewing altitude. The bending angle of L2 and the upper part of the bending angle profile of $\mathrm{L} 1$ is inverted using GO. The final profile is constructed by combining (sewing) the RH-based and GO-based profiles at an appropriate altitude (Kuo et al., 2004; S. Sokolovskiy and C. Rocken, personal communication, 2006). The sewing altitude of FSI-based and GO based profiles depends on strong fluctuation and abrupt noise increases in L2 Doppler, or large deviations between L1 and

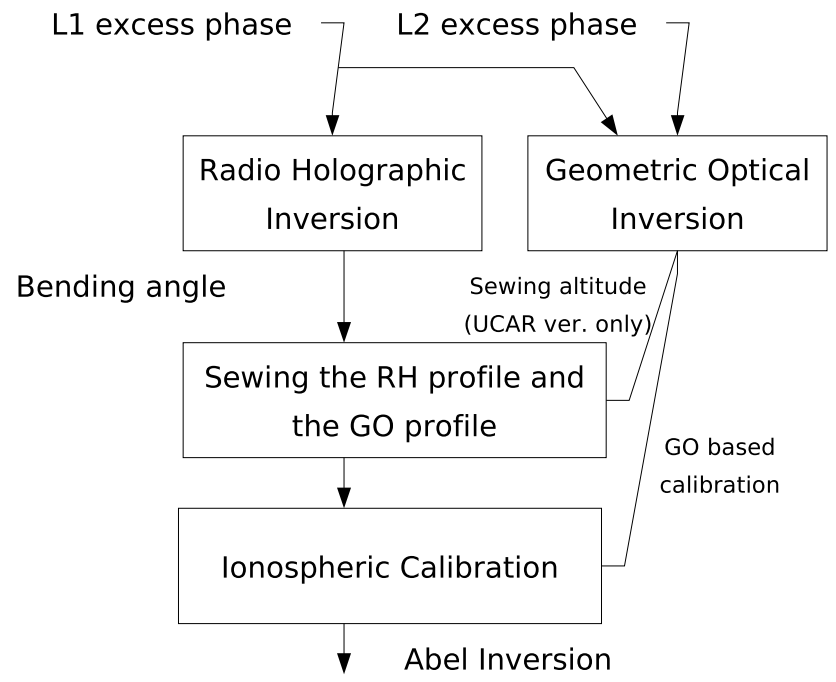

Fig. 1. Inversion scheme of GPS RO data by combining the GO and FSI methods.

L2 Doppler. Because CDAAC focuses on reducing multipath effects, especially below the tropopause, the sewing points are usually defined between 10 and $20 \mathrm{~km}$. However, atmospheric profiles with better vertical resolution are sometimes required for scientific and practical applications in meteorology, the atmospheric sciences, and so on. In this study, we use a different sewing scheme from CDAAC. Specifically, in our version the sewing altitude is much higher $(30 \mathrm{~km})$. Note that features such as optimization of the bending angle using a model profile, Abel inversion, and further derivation of atmospheric parameters are the same as with their counterparts in the GO inversion scheme.

Figure 1 shows a flowchart of the retrieval procedure used in this study, which is modified from that developed at CDAAC. L1 and L2 excess phases are the input of the GO inversion block. The L1 and L2 Doppler shifts are computed for a sewing criterion that outputs to the Sewing block, which combines the GO and RH based profile. Another output is L1 and L2 GO-based bending angles, which help calibrate the ionospheric effect in the combined bending angle profile. Note that because the RH method is only used below the sewing point, where the L2 signal is usually considered unavailable, ionospheric calibration for the RH-based profile is computed from extrapolation of the L2 bending angles. Ionospheric calibration in higher altitudes is calculated in the same manner as in the CDAAC version, while below $30 \mathrm{~km}$ the RH-based profile is corrected using the GO-based L1 and L2 bending angles. 


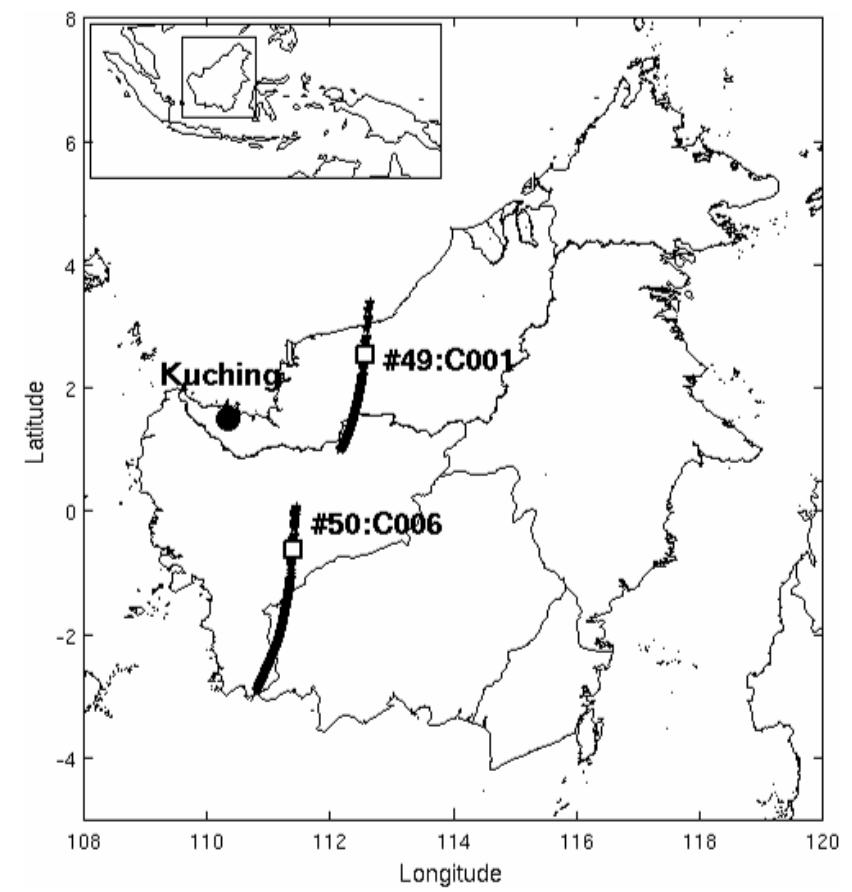

Fig. 2. Locations of tangent points for two COSMIC GPS RO events (\#49: C001 and \#50: C006) on 25 November 2006 near the radiosonde station at Kuching, Malaysia (solid circle). For both GPS RO events the tangent point moved from south to north. The south-end point and the square symbol correspond to $40 \mathrm{~km}$ and about $4 \mathrm{~km}$ altitude, respectively.

\section{Validation of GPS RO temperature profiles with radiosondes}

When the sewing height is elevated, it may introduce noise with short vertical scales from several error sources, such as ionospheric perturbations or receiver system noise, and such noise can contaminate the atmospheric excess phase. It is noteworthy that the atmospheric excess phase decreases exponentially with altitude, and thus noise effects become more serious at higher altitudes. Consequently, validation of retrieved results is particularly necessary at higher altitudes.

Here, we verify the FSI-based temperature profiles through comparison with radiosonde results with good height resolution. We obtained 54 radiosonde profiles launched at Kuching $\left(1.5^{\circ} \mathrm{N}, 110.4^{\circ} \mathrm{E}\right)$ by the Malaysian Meteorological Department. We analyzed the original data records of VAISALA radiosondes (type RS-80), and obtained a temperature profile with a height resolution of about $100 \mathrm{~m}$. Figure 2 shows locations of two GPS RO events, \#49 $\left(2.5^{\circ} \mathrm{N}\right.$, $\left.112.6^{\circ} \mathrm{E}, 10: 19 \mathrm{UTC}\right)$ and $\# 50\left(0.6^{\circ} \mathrm{S}, 111.4^{\circ} \mathrm{E}, 10: 32 \mathrm{UTC}\right)$ on 25 November 2006 (Day-Of-Year, 329). These RO events occurred within about one hour from the balloon launch at 11:39 UTC from Kuching, Malaysia, and the distance between the launch site and the RO tangent position at around $4 \mathrm{~km}$ altitude (square symbols in Fig. 2) was about $250 \mathrm{~km}$.

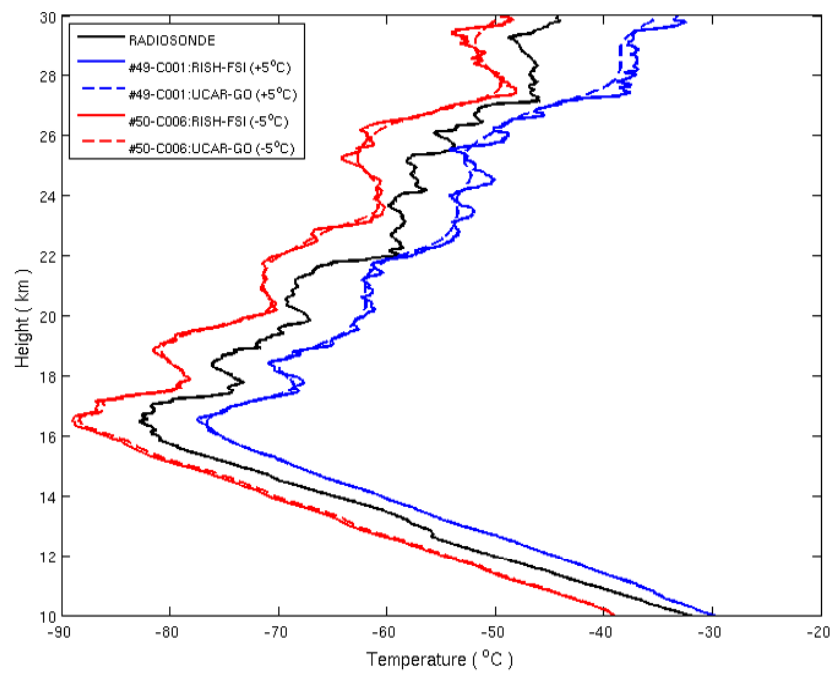

Fig. 3. Comparison of temperature profiles from the COSMIC GPS $\mathrm{RO}$ and radiosonde launched at Kuching (black line in the middle). The solid blue and red lines correspond to the FSI results analyzed in this study for \#49 and \#50, respectively, and the thin dashed lines are taken from the CDAAC database. The profiles are shifted by $+5 \mathrm{~K}$ and $-5 \mathrm{~K}$ for $\# 49$ and $\# 50$ relative to the radiosonde profile.

The temperature profiles are shown in Fig. 3. The solid lines in Fig. 3 are the FSI retrieval for \#49 and \#50 in our study, and the dashed lines are taken from CDAAC and analyzed with the GO method. Since the effect of humidity on the refractive index becomes negligible above the tropopause, located at about $16 \mathrm{~km}$, we use the dry temperature of GPS-RO.

In Fig. 3 the profiles above the tropopause exhibit smallscale temperature perturbations in the FSI and radiosonde profiles, while the GO profile shows a rather smooth height structure. A sharp change in the temperature gradient is clearly seen near the tropopause. In the stratosphere wavelike structures are evident at $18-20 \mathrm{~km}$ altitude, showing a downward phase progression from \#50 to Kuching and toward \#49. This can be interpreted as a manifestation of an atmospheric gravity wave that propagated northward over these sites. This example provides proof that the FSI method is sufficient to analyze temperature perturbations with vertical resolution comparable with a radiosonde, even in the stratosphere.

Next, for statistical validation we compare the temperature profiles between GPS-RO and high-resolution radiosondes in the United States provided by NOAA from October to December 2006. The radiosonde observations were made at 93 stations, 69 in the continental US, 13 in Alaska, 10 in the Pacific, and one in Puerto Rico. The GPS-RO events were selected by locating GPS RO tangent points whose distance from the radiosonde launch sites were less than $400 \mathrm{~km}$ and with time separation less than $30 \mathrm{~min}$. The number of compared pairs ranges from 210 to about 350, depending on the month and altitude range. 
Standard deviations and the mean temperature differences (GPS-RO minus radiosonde) are shown in Fig. 4 for the GO and FSI methods in November 2006. The mean absolute temperature difference at $10-30 \mathrm{~km}$ for FSI is less than $0.5 \mathrm{~K}$ for all three months. Note that the results are not shown for October and December, as the tendency is very similar to those in Fig. 4. There is no obvious temperature bias for either FSI or GO, and the accuracy of the FSI method seems to be similar to that of GO. The Standard deviation in Fig. 4 could mostly reflect natural variability, such as gravity waves, rather than error. Because profiles with radiosondes and GPS RO in Fig. 4 are obtained with certain time difference and spatial separation, temperature perturbations caused by gravity waves may have phase shift, which results in the standard deviation of temperature differences. The average standard deviation for FSI does not vary significantly with altitude. At high altitudes, where the number of comparisons is similar between the FSI and GO profiles, the standard deviation and the mean are very similar. At CDAAC most of the GO profiles are replaced with the FSI results below about $16 \mathrm{~km}$, so as the number of GO profiles decreases the number of comparisons below $16 \mathrm{~km}$ also decreases.

\section{Vertical wave number spectrum}

Here, we analyze vertical wave number spectra of temperature fluctuations, and compare them with a theoretical model and radiosonde observations. Through statistical investigation of the spectra, we aim at identifying the vertical resolution of FSI retrievals, and their dependence on the altitude region in the stratosphere.

First, we briefly introduce the fundamental concept of the vertical wave number spectrum of saturated gravity waves (Smith et al., 1987). Earlier studies reported that temperature profiles in the upper troposphere and the stratosphere fluctuate with vertical scales ranging from tens of meters to several kilometers. These fluctuations are believed to be superpositions of various atmospheric gravity waves with different wave lengths (e.g. Dewan, 1979; VanZandt, 1982). Spectral analysis is adopted to express gravity wave characteristics. For large vertical wave numbers (short wave lengths), the spectral energy density and the logarithmic spectral slope are nearly invariant, regardless of the altitude range and geographical location (VanZandt, 1982). Observational results indicate that the asymptotic logarithmic slope of the vertical wave number spectra is close to -3 for large wave numbers, while the spectral density is limited by the saturation values determined by the background Brunt-Vaisala frequency (Smith et al., 1987; Fritts et al., 1988; Tsuda et al., 1989). A formula for the saturated gravity wave spectrum for the normalized temperature perturbations can be expressed as follows for a large wave number $m$,

$$
F_{T^{\prime \prime} / T}(m)=N^{4} /\left(10 \mathrm{~g}^{2} \mathrm{~m}^{3}\right),
$$

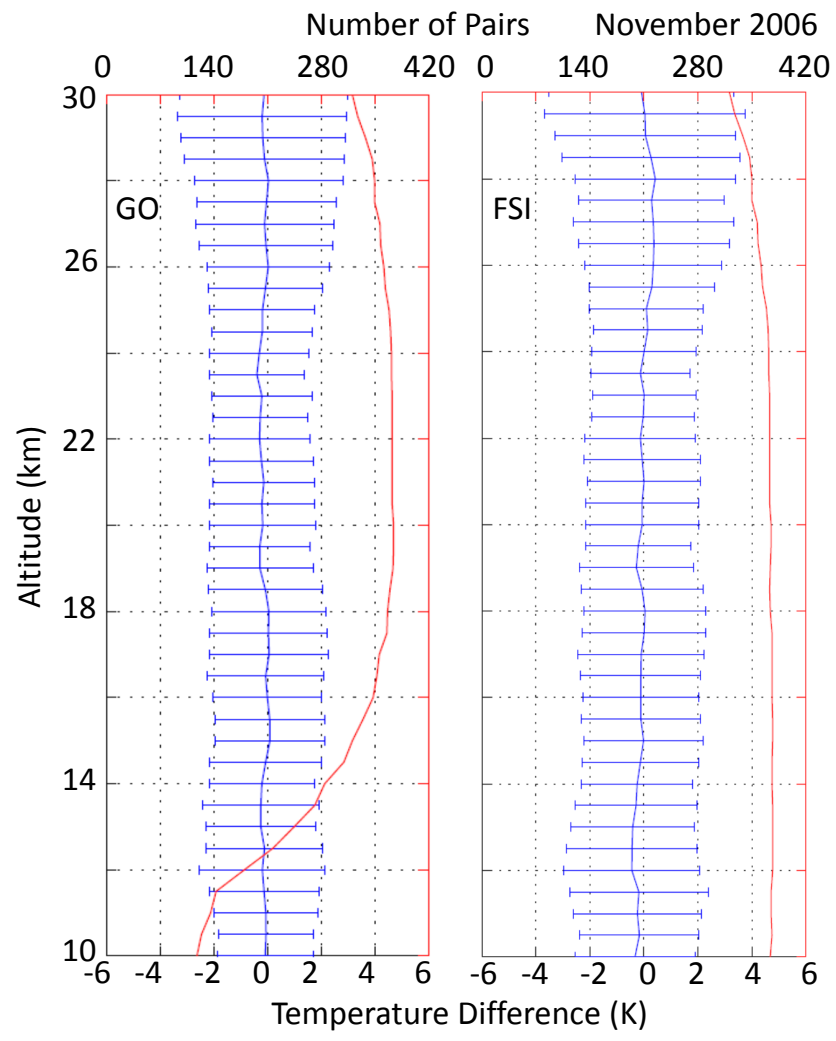

Fig. 4. Comparisons between COSMIC GPS RO profiles and high-resolution routine radiosonde data in November 2006 in North America. The blue lines show the temperature differences and standard deviations (bottom scale), where the GO and FSI methods are adopted for GPS RO retrieval in left and right panels, respectively. The red lines indicate the number of pairs for comparisons (top scale).

where $N$ is the Brunt-Vaisala frequency, $g$ is the acceleration of gravity and $T^{\prime}$ and $T$ are the temperature perturbation and the background temperature, respectively (Tsuda et al., 1991). This relation was validated by intensive radiosonde observations (Tsuda et al., 1994). It is noteworthy that Eq. (1) provides the maximum of the spectral density for saturated gravity waves because of wave dissipation due to convective instability (e.g. Fritts and Alexander, 2003).

The vertical wave number spectra were analyzed using GPS RO temperature profiles from GPS/MET, which demonstrated similar characteristics of the asymptotic logarithmic slope with a model spectrum of saturated gravity waves (Steiner and Kirchengast, 2000; Tsuda and Hocke, 2002). These results are based on GO retrieval, however, which may not fully resolve small vertical scales. In this study we analyze the vertical number spectra of temperature fluctuations using FSI results.

Figure 5 shows the spectra from GPS RO with both GO and FSI methods compared to the theoretical model. A spectrum from the US radiosonde data is also plotted. The 


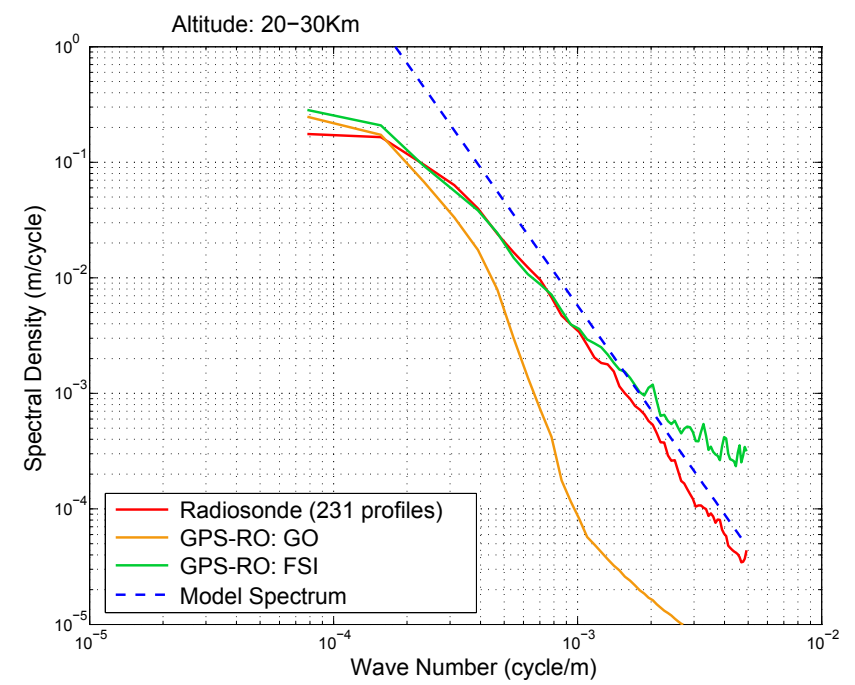

Fig. 5. Mean vertical wave number spectra of the normalized temperature perturbations at $20-30 \mathrm{~km}$ altitude in October-December 2006. The green and orange lines correspond to the spectra using the FSI and GO retrievals, respectively. The red line is an average spectrum from 231 radiosonde profiles obtained in North America. The dashed blue line indicates the model spectrum for large wave numbers with $N^{2}=4.67 \times 10^{-4}\left(\operatorname{rad}^{2} \mathrm{~s}^{-2}\right)$.

Brunt-Vaisala frequency $N$ is computed from the mean temperature and pressure profiles with radiosondes. (Note that the unit for $m$ in Fig. 5 is cycles $m^{-1}$ ). Spectra from both radiosonde and FSI in Fig. 5 are approximately parallel with the model except for the first few spectral points where gravity waves are not saturated, and therefore the spectrum does not follow Eq. (1).

The FSI spectrum closely matches that from radiosondes at wave numbers as large as about $2 \times 10^{-3}$ cycles $^{-1}$. Beyond $2 \times 10^{-3}$ cycles $\mathrm{m}^{-1}$, the FSI spectrum tends to show a gradual (flat) slope, and the spectral density becomes larger than the model values. This indicates the appearance of a noise floor for large $m$. The spectra retrieved by GO show a much smaller spectral density than the model, particularly for large $m$. This is caused by smoothing with a filter in the inversion program, which is adjusted to a height resolution of about $1.5 \mathrm{~km}$ by considering the Fresnel radius in the stratosphere.

For statistical validation, we calculated the spectra by averaging over all December 2006 COSMIC GPS RO profiles at $20-30 \mathrm{~km}$ in the latitude ranges $20^{\circ}-40^{\circ} \mathrm{S}$ and $20^{\circ}-40^{\circ} \mathrm{N}$. The results using both FSI and GO are shown in Fig. 6. The spectra from these two latitude bands show a very similar shape. The number of averaged profiles is much larger than in Fig. 5, making the spectra much smoother, but the noise floor does not disappear.

The noise floor is related to the vertical resolution of the FSI method. Because a bend from -3 to 0 (white noise) in the logarithmic spectral slope appears at around

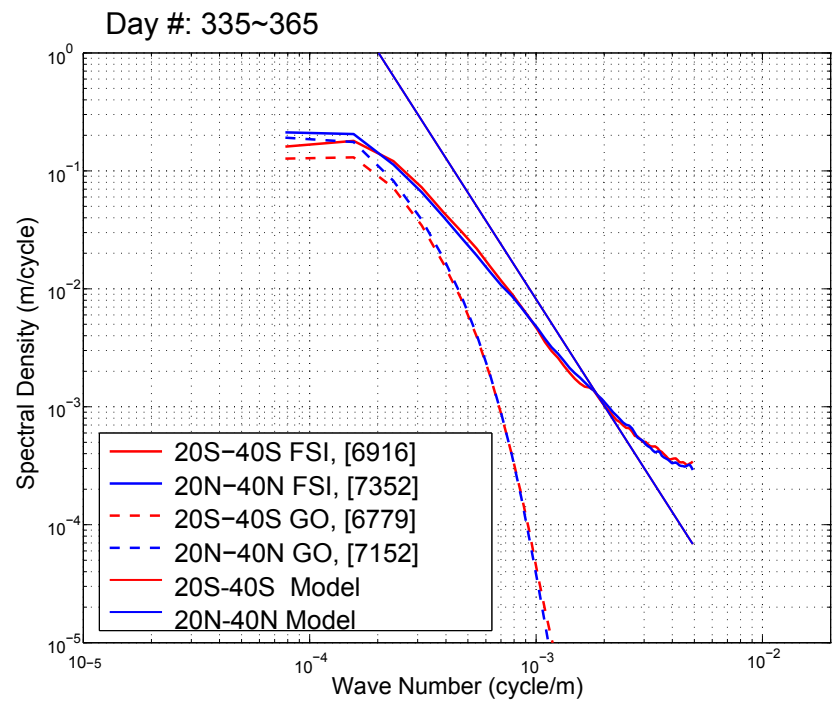

Fig. 6. Vertical wave number spectra from the COSMIC data in December 2006 at $20^{\circ}-40^{\circ} \mathrm{S}$ (red lines) and $20^{\circ}-40^{\circ} \mathrm{N}$ (blue lines). Solid and dashed lines correspond to the FSI and GO methods, respectively. The number of GPS RO profiles used to calculate the mean spectra is shown in parentheses. The straight line indicates the model spectrum with $N^{2}=5.57 \times 10^{-4}\left(\mathrm{rad}^{2} \mathrm{~s}^{-2}\right)$.

$3 \times 10^{-3}$ cycles $\mathrm{m}^{-1}$, we could resolve waves with a vertical wave length of about $300 \mathrm{~m}$. The vertical resolution of the FSI retrieval seems to be about $150 \mathrm{~m}$, i.e. half the shortest detectable wave length.

Earlier studies estimated the vertical resolution of RH methods, including FSI, using numerical simulations. Mortensen et al. (1999) showed the resolution to be about $100 \mathrm{~m}$, while Gorbunov et al. (2004) indicated a better resolution of about $60 \mathrm{~m}$. Those studies, however, assumed an ideal case without additional errors such as ionospheric noise or horizontal inhomogeneities, thus giving inferred vertical resolutions better than our estimate based on observed results.

It is important to note that vertical resolution can also vary with altitude. Figure $7 \mathrm{a}$ and $\mathrm{b}$ shows the spectra in three altitude ranges, $17-24 \mathrm{~km}, 20-27 \mathrm{~km}$, and $23-30 \mathrm{~km}$, at (a) mid-latitudes $\left(20^{\circ}-40^{\circ} \mathrm{S}\right)$ and (b) equatorial regions $\left(20^{\circ} \mathrm{S}-20^{\circ} \mathrm{N}\right)$, respectively. The noise floor magnitude increases with altitude, and is most evident at $23-30 \mathrm{~km}$ in Fig. 7. This suggests that the noise floor in the spectra at 20$30 \mathrm{~km}$ (see Fig. 6) could be mainly attributable to the high altitude region.

\section{Upper limit of the FSI profiles}

The atmospheric excess phase decreases exponentially with altitude, as it is basically related to atmospheric density. System noise may not depend on altitude, but ionospheric effects increase at higher altitudes. The quality of the FSI profile, therefore, tends to degrade at higher altitudes. The main 
purpose here is to define the maximum available height of FSI profiles for studies of small vertical scale geophysical perturbations such as atmospheric gravity waves.

We utilize the behavior of the noise floor in the vertical wave number spectra as an index of deterioration of the FSI retrieval. We analyze the wave number spectra for individual GPS RO profiles using the same procedure as in Figs. 5-7, but shorten the height range for spectral analysis from $7-10 \mathrm{~km}$ to $2 \mathrm{~km}$ and concentrate on the behavior of the spectra at large $\left(>10^{-3}\right.$ cycles $\left.\mathrm{m}^{-1}\right)$ wave numbers. If the spectral density noticeably exceeds the model value given in Eq. (1), then the origin of the temperature perturbations are most likely not gravity waves, but they are affected by system noise and ionospheric effects. We frequently found that the spectra at $26-28 \mathrm{~km}$ and $28-30 \mathrm{~km}$ altitudes show noisy behavior, with the spectral density obviously exceeding theoretical predictions.

For the Fourier Integral Operator (FIO) method including FSI, Gorbunov et al. (2006) proposed a technique that can estimate signal tracking errors for bending angles. This technique is based on the width of the running spectra of the transformed wave field multiplied by a reference signal. The reference signal is computed by smoothing the phase of the transformed field. In this method, however, the smoothing parameter should match the vertical scale of the noise. In our case, noise may have various wave lengths in different profiles, and thus it is difficult to use a constant smoothing parameter for all measurements.

Lohmann (2006) proposed another error estimation for bending angles based on fluctuations in the log amplitude of the Fourier transformed signals. This method can work on each profile without external data other than a signal-to-noise ratio (SNR) threshold. In the standard inversion program at CDAAC, estimated errors of the bending angle are calculated using this method. But this estimation does not distinguish perturbations with different wave lengths.

Here we propose a simple procedure concentrating on the fluctuation with different wave lengths in the bending angle profiles, and then determine the upper limit for the FSI profile. Considering saturated gravity wave theory, the normalized temperature perturbations $T^{\prime} / T_{0}$ should not exceed a certain threshold. Similarly, the ratio of the bending angle perturbation $\alpha^{\prime}$ to $\alpha_{0}$, the optimized bending angle with GO, can be limited by a threshold. Provided the bending angle perturbation caused by system noise remains the same magnitude throughout an occultation event, the ratio $\alpha^{\prime} / \alpha_{0}$ exponentially increases with altitude. We therefore assume that the height variation of the ratio $\alpha^{\prime} / \alpha_{\mathrm{o}}$ can be used as an index to judge contamination by noise. When noise dominates the ratio increases exponentially with altitude, but it remains nearly constant when the signal instead comes from actual atmospheric perturbations.

Figure 8 shows an example of $\alpha^{\prime} / \alpha_{\mathrm{o}}$ versus height for the profile C001.2006.335.00.50.G23. We consider two vertical scale ranges, $0.1-0.5 \mathrm{~km}$ and $1-2 \mathrm{~km}$, and estimate $\alpha^{\prime}$ from
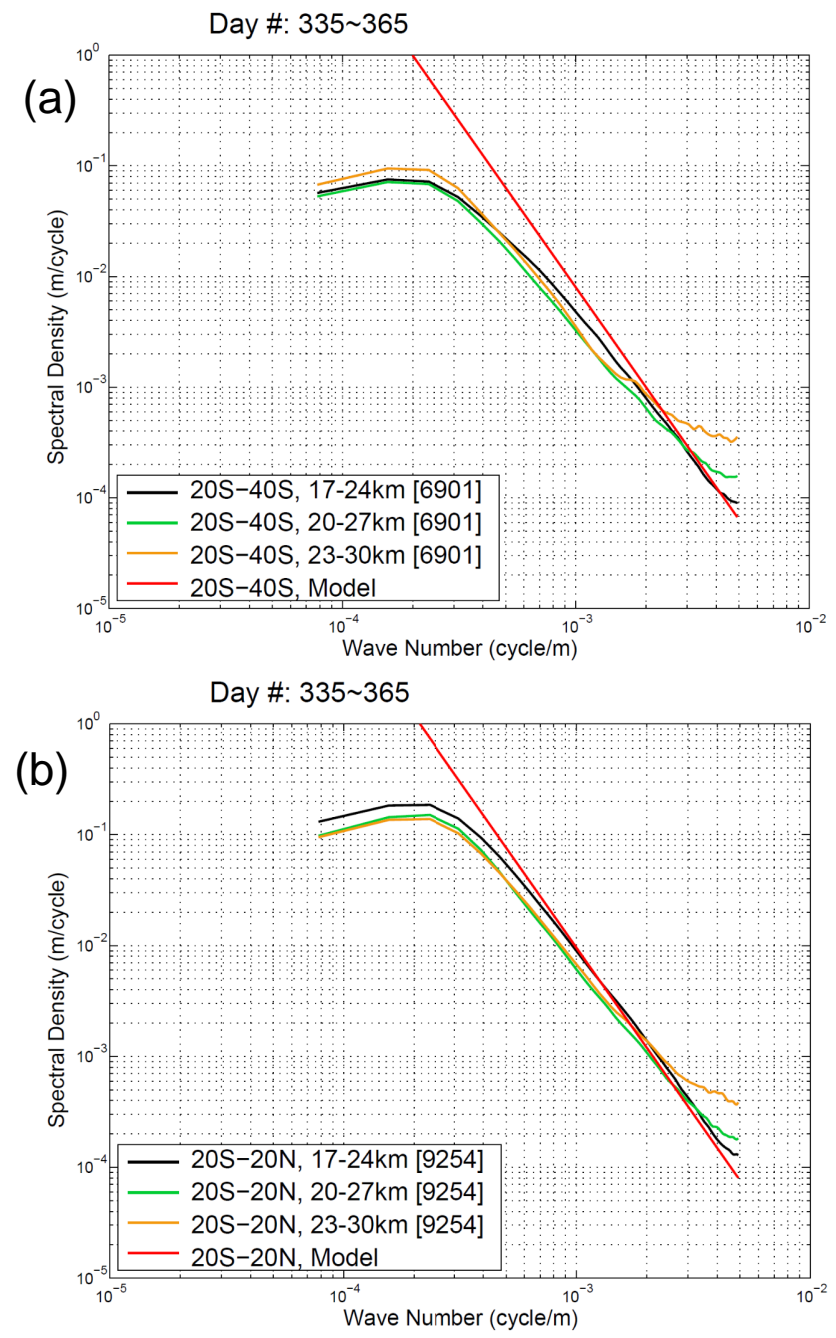

Fig. 7. Vertical wave number spectra using the FSI method at (a) $20^{\circ}-40^{\circ} \mathrm{S}$ and (b) $20^{\circ} \mathrm{S}-20^{\circ} \mathrm{N}$ in altitude ranges of $17-24 \mathrm{~km}$ (blue), 20-27 km (green), and 23-30 km (orange). The red line shows the model spectrum with $N^{2}=5.50 \times 10^{-4}\left(\mathrm{rad}^{2} \mathrm{~s}^{-2}\right)$ and $6.02 \times 10^{-4}\left(\mathrm{rad}^{2} \mathrm{~s}^{-2}\right)$ for (a) and (b), respectively.

the standard deviation of bending angle perturbations at each scale range. The ratio for short vertical scales rapidly increases above about $26 \mathrm{~km}$, but it is less than about $1 \%$ for longer scales in the entire height range. For this particular case, we should limit the maximum height to $26 \mathrm{~km}$ to study small-scale atmospheric perturbations.

According to Jensen et al. (2003), the accuracy of FSI is $1 \mathrm{~K}$. We take this value to infer a threshold. Considering a standard atmospheric model, like MSISE, the maximum bending angle fluctuation corresponding to the temperature perturbation with $1 \mathrm{~K}$ amplitude and vertical wave length of $0.5 \mathrm{~km}$ becomes about $2.1 \%$. For the estimate of standard deviation, we take $1.4 \%$ as the root mean square value of the sinusoidal temperature perturbations. 


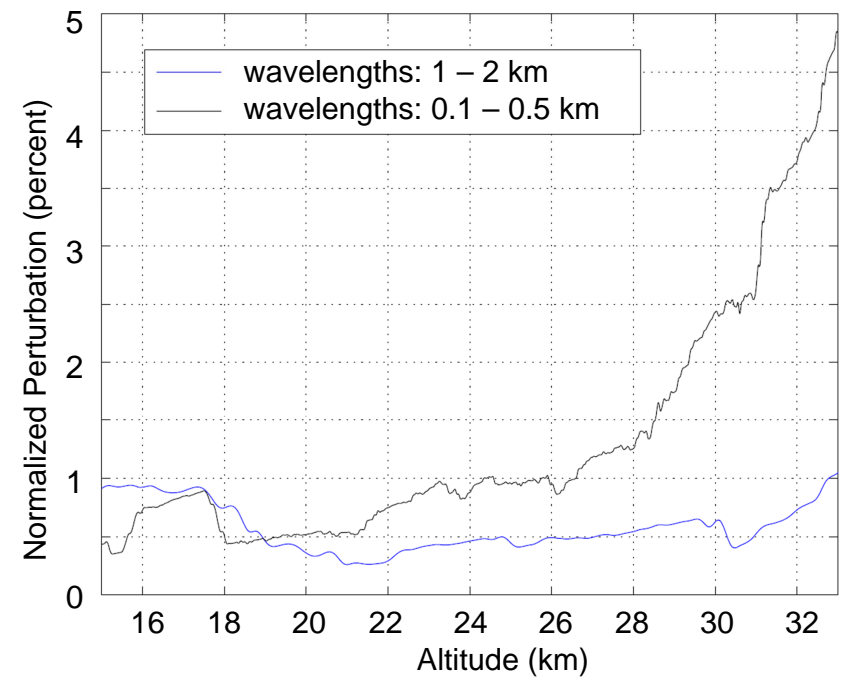

Fig. 8. Height variations of normalized fluctuations of the bending angle for height scales of $1-2 \mathrm{~km}$ (blue line) and $0.1-0.5 \mathrm{~km}$ (black line).

We statistically analyzed the distribution of the upper boundary in terms of local time at latitudes $20^{\circ} \mathrm{S}-20^{\circ} \mathrm{N}$, where ionospheric noises seem to be larger. We show the results for November and December 2006 in Fig. 9a and b, respectively, as the amount of GPS RO data fluctuates depending on local time. We only focus on the local hours where numerous profiles existed, specifically ranges 05:00-16:00 local time in November, and 09:00-14:00 and 21:00-03:00 local time in December. Note that longitudinal asymmetry of the ionosphere is greatest at sunrise and sunset. There is little difference between the two cases, however, suggesting that ionospheric effects are not significant in determining the upper limit of FSI retrieval. To summarize, Fig. 9 indicates that FSI profiles can be used up to about $28 \mathrm{~km}$ when studying temperature perturbations with vertical wave lengths as short as about $0.5 \mathrm{~km}$.

\section{Concluding remarks}

Because of its advantages in vertical resolution, accuracy, and self-calibration, the GPS RO technique is promising for areas such as the atmospheric and ionospheric sciences, global and mesoscale weather forecasting, climate change monitoring, and boundary layer research. Among the various retrieval algorithms for GPS-RO, the GO and FSI methods are commonly used. Because of limitations due to the Fresnel radius for GO, its vertical resolution is limited to about $1.5 \mathrm{~km}$ in the lower stratosphere, while FSI is not affected by such limitations.

We applied FSI up to $30 \mathrm{~km}$ on COSMIC data and obtained temperature profiles with a better vertical resolution than those published by CDAAC with GO. We compared

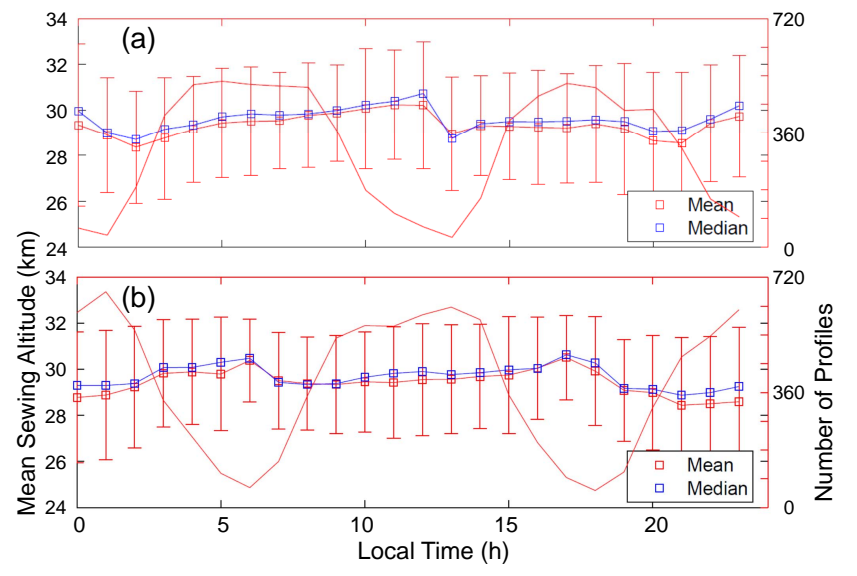

Fig. 9. Local time distribution of the mean (red square) and (blue square) median sewing altitude (left scale) together with the standard deviation at $20^{\circ} \mathrm{S}-20^{\circ} \mathrm{N}$ in (a) November 2006 and (b) December 2006. The red line indicates the number of profiles (right scale).

the FSI profiles with radiosonde obtained in Malaysia, which showed good consistency with perturbations caused by atmospheric waves. Further comparisons were done with North American radiosonde data from October to December 2006. Statistical tests indicate that the temperature errors for FSI are less than $0.5 \mathrm{~K}$, and the temperature bias is equivalent to GO retrieval.

We studied the vertical resolution of small-scale temperature fluctuations using a vertical wave number spectrum. Spectral analysis showed that a noise floor tends to appear for wave numbers larger than about $2-3 \times 10^{-3}$ cycles $^{-1}$. The vertical resolution of the FSI method in the lower stratosphere is inferred to be about $100-200 \mathrm{~m}$.

Since the atmospheric excess phase decreases exponentially with altitude, various forms of noise can easily contaminate GPS-RO retrieved data for high altitudes. A case study also showed noisy fluctuations in the bending angle profile above about $26 \mathrm{~km}$ altitude. We aimed at defining the upper limit for FSI profiles. By separating perturbations of the bending angle at different wave lengths, we found that smallscale perturbations are more sensitive to noise. We thus used a normalized perturbation of the bending angle as an index to determine the upper boundary. Assuming a temperature precision of $1 \mathrm{~K}$, the upper limit for the available profiles is estimated to be about $28 \mathrm{~km}$. The distribution of the upper boundary does not seem to be affected by ionospheric conditions.

Although FSI retrieval provides a height resolution in the lower stratosphere (below about $28 \mathrm{~km}$ ) superior to other satellite measurements, horizontal resolution is not significantly improved. GPS RO therefore observes only part of the horizontal wave number spectra of temperature perturbations, and so the effects of an observational filter must be 
considered in interpreting the analyzed characteristics of atmospheric waves (e.g. Alexander, 1998; Preusse et al., 2008).

Acknowledgements. We deeply appreciate UCAR for their generous support in providing us with the FSI software package. This work was supported by Grant-in-Aid for Scientific Research (B) (21340138), Japan Society for the Promotion of Science (JSPS). We are grateful to the Malaysian Meteorological Department for providing us with the radiosonde data.

Edited by: A. K. Steiner

\section{References}

Alexander, M. J.: Interpretation of observed climatological patterns in stratospheric gravity wave variance, J. Geophys. Res., 96, 8627-8640, 1998.

Anthes, R. A., Ector, D., Hunt, D. C., Kuo, Y.-H., Rocken, C., Schreiner, W. S., Sokolovskiy, S. V., Syndergaard, S., Wee, T.K., Zeng, Z., Bernhardt, P. A., Dymond, K. F., Chen, Y., Liu, H., Manning, K., Randel, W. J., Trenberth, K. E., Cucurull, L., Healy, S. B., Ho, S.-P., McCormick, C., Meehan, T. K., Thompson, D. C., and Yen, N. L.: The COSMIC/FORMOSAT3 Mission: Early Results, B. Am. Meteorol. Soc., 89, 313-333, doi:10.1175/BAMS-89-3-313, 2008.

Dewan, E. M.: Stratospheric Waves Spectra Resembling Turbulence, Science, 204, 832-835, 1979.

Fritts, D. C. and Alexander, M. J.: Gravity wave dynamics and effects in the middle atmosphere, Rev. Geophys., 41, 1003, doi:10.1029/2001RG000106, 2003.

Fritts, D. C., Tsuda, T., Sato, T., Fukao, S., and Kato, S.: Observational Evidence of a Saturated GravityWave Spectrum in the Troposphere and lower Stratosphere, J. Atmos. Sci., 45, 17411759, 1988.

Gorbunov, M., Gurvich, A., and Bengtsson, L.: Advanced algorithms of inversion of GPS/MET satellite data and their application to reconstruction of temperature and humidity, Tech. Rep. 211, Max-Planck-Institute for Meteorology, Hamburg, 1996.

Gorbunov, M. A.: Canonical transform method for processing radio occultation data in lower troposphere, Radio Sci., 37, 1040, doi:10.1029/2000RS002592, 2002.

Gorbunov, M. E., Benzon, H.-H., Jensen, A. S., Lohmann, M. S., and Nielsen, A. S.: Comparative analysis of radio occultation processing approaches based on Fourier integral operators, Radio Sci., 39, RS6004, doi:10.1029/2003RS002916, 2004.

Gorbunov, M. E., Lauritsen, K. B., Rhodin, A., Tomassini, M., and Kornblueh, L.: Radio holographic filtering, error estimation, and quality control of radio occultation data, J. Geophys. Res., 111, D10105, doi:10.1029/2005JD006427, 2006.

Hajj, G. A., Ao, C. O., Iijima, B. A., Kuang D., Kursinski, E. R., Mannucci, A. J., Meehan, T. K., Romans, L. J., de la Torre Juarez, M., and Yunck, T. P.: CHAMP and SAC-C atmospheric occultation results and intercomparisons, J. Geophys. Res., 109, D06109, doi:10.1029/2003JD003909, 2004.

Horinouchi, T. and Tsuda, T.: Spatial structures and statistics of atmospheric gravity waves derived using a heuristic vertical crosssection extraction from COSMIC GPS radio occultation data, J. Geophys. Res., 114, D16110, doi:10.1029/2008JD011068, 2009.
Jensen, A. S., Lohmann, M. S., Benzon, H.-H., and Nielsen, A. S.: Full Spectrum Inversion of radio occultation signals, Radio Sci., 38, 1040, doi:10.1029/2002RS002763, 2003.

Karayel, E. T. and Hinson, D. P.: Sub-fresnel-scale vertical resolution in atmospheric profiles from radio occultation, Radio Sci., 32, 411-423, 1997.

Kuo, Y.-H., Wee, T.-K., Sokolovskiy, S., Rocken, C., Schreiner, W., Hunt, D., and Anthes, R. A.: Inversion and Error Estimation of GPS Radio Occultation Data, J. Meteorol. Soc. Japan, 82, 507531, 2004.

Kursinski, E., Hajj, G., Bertiger, W. I., Leroy, S. S., Meehan, T., Romans, L., Schofield, J., McCleese, D., Melbourne, W., Thornton, C., Yunck, T., Eyre, J., and Nagatani, R.: Initial results of radio occultation observations of Earth's atmosphere using the Global Positioning System, Science, 271, 1107-1110, 1996.

Kursinski, E., Hajj, G., Hardy, K., Schofield, J., and Linfield, R.: Observing Earth's atmosphere with radio occultation measurementes using GPS, J. Geophy. Res., 102, 429, doi:10.1029/97JD01569, 1997.

Lohmann, M. S.: Dynamic error estimation for radio occultation bending angles retrieved by the full spectrum inversion technique, Radio Sci., 41, RS5005, doi:10.1029/2005RS003396, 2006.

McDonald, A. J., Tan, B., and Chu, X.: Role of gravity waves in the spatial and temporal variability of stratospheric temperature measured by COSMIC/FORMOSAT 3 and Rayleigh lidar observations, J. Geophys. Res., 115, D19128, doi:10.1029/2009JD013658, 2010.

Melbourne, W.-G.: Radio Occultations using earth satellites, JPL Deep Space Communications and Navigation Series, WILEY, 2004.

Mortensen, M. D., Linfield, R. P., and Kursinski, E. R.: Vertical resolution approaching $100 \mathrm{~m}$ for GPS occultations of the Earth's atmosphere, Radio Sci., 34, 1475-1484, 1999.

Preusse, P., Eckermann, S. D., and Ern, M.: Transparency of the atmosphere to short horizontal wavelength gravity waves, J. Geophys. Res., 113, D24104, doi:10.1029/2007JD009682, 2008.

Seko, H., Shoji, Y., Kunii, M., and Aoyama, Y.: Impact of the CHAMP Occultation Data on the Rainfall Forecast, Data Assimilation for Atmospheric, Oceanic and Hydrologic Applications, edited by: Park, S. K. and Xu, L., Springer Berlin Heidelberg, 197-217, 2009.

Smith, S. A., Fritts, D. C., and VanZandt, T. E.: Evidence of a saturation spectrum of atmospheric waves, J. Atmos. Sci., 44, 1404-1410, 1987.

Sokolovskiy, S. V.: Modeling and inverting radio occultation signals in the moist troposphere, Radio Sci., 36, 441, doi:10.1029/1999RS002273, 2001.

Sokolovskiy, S. and Rocken, C.: Algorithms for inverting radio occultation signals in the neutral atmosphere, University Corporation for Atmospheric Research, 2006.

Steiner, A. K. and Kirchengast, G.: Gravity wave spectra from GPS/MET occultation observations, J. Atmos. Ocean. Tech., 17, 495-503, 2000.

Tsuda, T. and Hocke, K.: Vertical Wave Number Spectrum of Temperature Fluctuations in the Stratosphere using GPS Occultation Data, J. Meteorol. Soc. Japan, 80, 925-938, 2002.

Tsuda, T., Inoue, T., Fritts, D. C. , VanZandt, T. E., Kato, S., Sato, T., and Fukao, S.: MST radar observations of a saturated gravity 
wave spectrum, J. Atmos. Sci., 46, 2440-2447, 1989.

Tsuda, T., VanZandt, T. E., Mizumoto, M., Kato, S., and Fukao, S.: Spectral Analysis of Temperature and Brunt-Vaisala Frequency Fluctuations Observed by Radiosondes, J. Geophys. Res., 96, 17265-17278, doi:10/1029.91JD01944, 1991.

Tsuda, T., Murayama, Y., Wiryosumarto, H., Harijiono, S. W. B., and Kato, S.: Radiosonde Obervations of Equatorial Atmosphere Dynamics over Indonesia, J. Geophy. Res., 99, 10507-10516, 1994.

Tsuda, T., Nishida, M., Rocken, C., and Ware, R. H.: A Global Morphology of Gravity Wave Activity in the Stratosphere Revealed by the GPS Occultation Data GPS/MET, J. Geophys. Res., 105(D6), 7257-7273, 2000.
VanZandt, T. E.: A Universal Spectrum of Buoyancy waves in the Atmosphere, Geophys. Res. Lett., 9, 575-578, 1982.

Ware, R., Exner, M. , Feng, D., Gorbunov, M., Hardy, K., Herman, B., Kuo, Y., Meehan, T., Melbourne, W., Rocken, C., Schreiner, W., Sokolovskiy, S., Solheim, F., Zou, X., Anthes, R., Businger, S., and Trenberth, K.: GPS sounding of the atmosphere From Low Earth Orbit: Preliminary Results, B. Am. Meteorol. Soc., 77, 19-40, 1996.

Wickert, J., Reigber, C., Beyerle, G., Koenig, R., Marquardt, C., Schmidt, T., Grunwald, L., Galas, R., Meehan, T. K., Melbourne, W. G., and Hocke, K.: Atmospheric soundings by GPS radio occultation: First results from CHAMP, Geophys. Res. Lett., 28, 3263-3266, 2001. 